Kubler-Ross analysis. The next three chapters are the book's kernel, covering ethical issues, legal aspects and economic considerations. Inevitably the transatlantic origin is apparent here, but the analysis of legal provisions in different states provides an excellent overview of their implications and is easy to follow because each is preceded by an unemotional statement of the facts of specific cases which led to litigation. The final chapter, When it happens to you, attempts to provide practical as well as emotional help.

A special feature of the book is that each chapter opens with a case history, reproduced in bold type which is temptingly easy to read in isolation. It is to your disadvantage to do so - unlike the legal illustrations mentioned earlier, these examples are often well chosen but are presented in emotive prose (chapter 3) or with stereotyped characters in whom it is difficult to believe (chapter 11). The text outlining how a practitioner advised a patient and family against intensive care (chapter 7) describes an uninterrupted monologue which carries little conviction that this is how such a difficult matter is, or should be, handled.

This leads to the most fundamental criticism of the book - these case histories and much of the first half are written in a style which is either irritatingly trite or simple to the point of patronage. Euphemisms such as 'peaceful departure' are interspersed with 'to pull the plug' (even suggesting that this is carried out by a respiratory therapist); similarly 'in restraints to protect his intravenous line' or 'tied down'.

Those who wish to explore the issues covered in the second half of the book still in simple and lucid prose - may well find the first half irksome and might prefer a style which avoided the emotive prose more usually associated with the less responsible components of our media. If you can bear with it, the rewards are all at the end where the last four chapters make fine reading.

M A BRANTHWAITE Consultant Physician

Brompton Hospital Fulham Road

London SW3 6HP

\section{Life and Death Decision Making}

Baruch Brody, 250 pages, USA, £22.50, Oxford University Press, 1988.
This book is a sincere, worthy and on the whole successful attempt to assess the pros and cons of 'Life and death' decisions, made almost exclusively within the setting of an intensive care unit in the United States. The author describes the need for a 'moral theory' and one must, of course, agree with this. However, most people without the gift of religious faith and revelation can perceive no simple way of arriving at a moral theory, since the moral infrastructure of society is not static over time. However, the author makes a heroic effort to identify various ethical 'appeals' which have to be considered in every difficult decision, ie 'respect for persons', 'cost-effectiveness and justice', 'rights', 'consequences of our actions', and 'the virtues'.

The author concludes that none of the current ethical systems take account of all these 'appeals'. After discussing some of the other guidelines which have been proposed he itemises a new model for the patient-physician relationship which takes account of the various 'appeals' which frequently conflict and whose strength has to be assessed positively and negatively.

I found this first part of the book sensible and there was little that one would disagree with. I was unsure to what extent any original points were made. However, I found the last two thirds of the book quite fascinating. These comprise a detailed account of 40 very realistic clinical situations which covered the care of elderly and frequently fatally ill patients, young children, and neonates with serious medical disorders, as well as some young adults. Some of the situations envisaged seem almost unreal to a British physician because of the high level of public awareness in the United States that bodies can be kept 'alive' with the heart continuing to beat and ventilation maintained artificially for virtually unlimited periods. Some of the 'decision-making' seems hardly worth discussing. For example, three pages are devoted to the process of arriving at the conclusion that a baby born with hydranencephaly should not be aggressively managed. In general, I found no difficulty in agreeing with the conclusions of the author and his reviewing panel in most of the clinical situations discussed, although I felt in many cases that unduly heavy weather was being made. However, I found it very difficult to agree that a previously healthy 84-year-old woman who had broken her leg and was refusing to eat should be force-fed if persuasion failed. I found this suggestion almost obscene but I recognise that this sort of situation is an ethical gray area and I suspect that physicians in the United States are somewhat more therapeutically aggressive in the elderly than their counterparts in Britain.

To summarise, I felt this was a worthy attempt to clarify ethical issues in life and death situations and the case analyses that comprise the latter two thirds of the book could be read with advantage by all medical students and any doctors who face some of the difficult decisions which are discussed and analysed.

PROFESSOR C J DICKINSON Professor of Medicine and Chairman, Academic Department of Medicine, St Bartholomew's Hospital Medical College, London

\section{Logic in Medicine}

Edited by Calbert I Phillips, 104 pages, London, £5.95, British Medical fournal, 1988.

Logic in Medicine is captivating in its clarity and offers a challenge to those who believe that medicine is more an art than a complex science governed by logic. On the other hand, those who believe that medicine is respectable and credible to the extent that it can justify its claims by careful reasoning will find this book a cogent illustration of that position. The book consists of six articles each of which focusses on the use and value of systematic reasoning in a particular area of medical theory and decision-making. The chapters discuss the following topics: doctors and witchcraft, formal logic, diagnostic logic, diagnostic systems as an aid to clinical decision-making, an economic perspective showing the use of logic in allocation decisions, and finally, an argument for fundamental ethical principles in health care.

The cumulative effect of reading this book is the renewed realisation that an essential component in the practice of medicine is the conscious use of method and reasoning. This attention to greater rigour in method is recommended as a necessary supplement to an intuitive approach based on experience which 'usually serves remarkably well' ( $p$ ix). However, an emphasis on reflective method does not promise certainty either in diagnosis or treatment decisions. In an excellent discussion of diagnostic logic, Fergus Macartney rejects the need for certainty in diagnosis in the general management of 